\title{
Pseudo-zero-mode Landau levels and pseudospin waves in bilayer graphene
}

\author{
K. Shizuya
}

Yukawa Institute for Theoretical Physics, Kyoto University, Kyoto 606-8502, Japan

\begin{abstract}
Bilayer graphene in a magnetic field supports eight zero-energy Landau levels, which, as a tunable band gap develops, evolve into two nearly-degenerate quartets separated by the band gap. A close look is made into the properties of such an isolated quartet of pseudo-zero-mode levels at half filling in the presence of an in-plane electric field and the Coulomb interaction. The pseudo-zeromode levels turn out to support, via orbital mixing, charge carriers with induced electric dipole moment, which lead to characteristic collective excitations, pseudospin waves, with some controllable features; possible experimental signals are discussed.
\end{abstract}

'Key words: Graphene, bilayer, quantum Hall effect, collective excitations

\section{Introduction}

Graphene attracts a great deal of attention, both experimentally and theoretically [1, 2], for its exotic electronic transport. Of particular interest is bilayer graphene [3], which has a unique property that the (conduction-valence) band gap is controllable [4] by use of external gates or chemical doping.

A particle-hole symmetric tower of Landau levels and the presence of some zero-energy levels are features specific to graphene in a magnetic field. Bilayer graphene supports eight such zero-energy levels, which, as a tunable band gap develops, evolve into two nearly-degenerate quartets separated by the band gap.

In this paper we would like to report some unusual properties of such an isolated quartet of pseudo-zero-mode levels, especially, coherence and collective excitations in the presence of an in-plane electric field $\mathbf{E}_{\|}$and the Coulomb interaction [5]. We focus on revealing further controllable features in bilayer graphene and point out the following:

(1) The pseudo-zero-mode levels, especially at half filling, support, via orbital level mixing, charge carriers with a nonzero 'electric dipole moment. As a result, fine splitting of the pseudozero-mode quartet is also externally controlled by an in-plane 'electric field or by an injected Hall current.

(2) The interplay of the Coulomb interaction and an external field leads to rich spectra of collective excitations, (orbital) pseudospin waves.

\section{Pseudo-zero-mode levels and dynamics}

Monolayer graphene supports as charge carriers massless Dirac fermions with a linear dispersion. In bilayer graphene interlayer coupling modifies the intralayer "relativistic" spectra to yield, in the low-energy branches, massive quasiparticles[3], which, in a magnetic field $B$, lead to a tower of Landau levels $\left|n, y_{0}\right\rangle$ of energy

$$
\epsilon_{n}=\operatorname{sgn}[n] \omega_{\mathrm{c}}^{\mathrm{bi}} \sqrt{|n|(|n|-1)},
$$

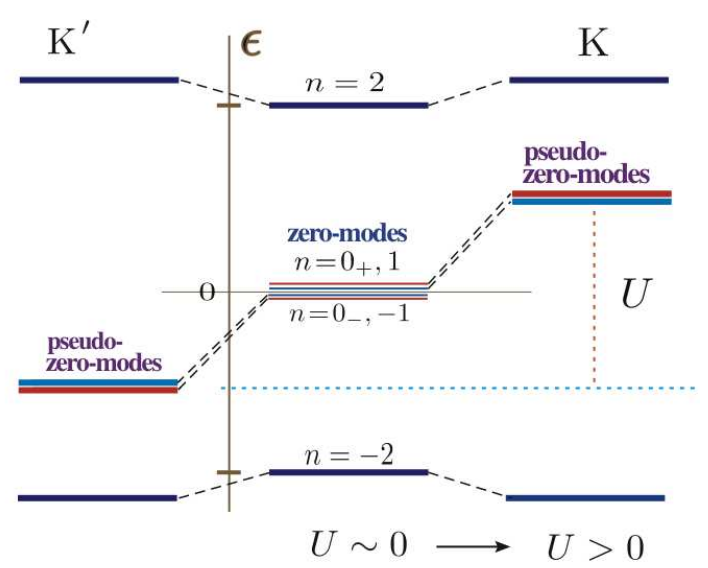

Figure 1: Pseudo-zero-mode Landau levels

labeled by integers $n= \pm 0, \pm 1, \pm 2, \ldots$, the center coordinate $y_{0} \equiv \ell^{2} p_{x}$ with the magnetic length $\ell=1 / \sqrt{e B}$ and the characteristic cyclotron energy $\omega_{c}^{\mathrm{bi}} \sim 4 \times B[\mathrm{~T}] \mathrm{meV}$ (with $B$ in tesla).

The $|n|=0$ and $|n|=1$ levels have zero energy, and, with a band gap $U>0$, evolve into two sectors of nearly-degenerate pseudo-zero-mode levels (separated by a band gap and in valley), with spectra

$$
\epsilon_{n= \pm 0}= \pm \frac{1}{2} U, \epsilon_{n= \pm 1}= \pm\left(\frac{1}{2} U-2 m\right)
$$

where $m \approx 0.006 \times B[\mathrm{~T}] U \ll U$ denotes a fine splitting within each sector.

The presence of the zero-energy levels and their double-fold degeneracy (per spin and valley) have a topological origin. This degeneracy is affected by a band gap $U$ and an external field $A_{0}$. Accordingly, the pseudo-zero-mode levels have an intrinsic tendency to be degenerate, but its fine structure depends sensitively on the environment.

The purpose of the present paper is to study such controllable features of the pseudo-zero-mode quartet in the presence of external fields and Coulomb interactions. We are particularly 
interested in the properties of this quartet at half filling, where mixing of the pseudo-zero-modes may take place. For definiteness we focus on the $n=\left(0_{+}, 1\right)$ sector at one valley, i.e., around filling factor $v=2$ (or $v=1$ when the spin is resolved), and ignore the presence of other levels which are separated by relatively large gaps.

The pseudo-zero-mode levels of our interest contain two different Landau orbitals $n=0_{+}$and $n=1$ (per spin). Let us put these modes, $\psi_{0_{+}}\left(y_{0}, t\right)$ and $\psi_{1}\left(y_{0}, t\right)$, into a two-component spinor $\Psi=\left(\psi_{0_{+}}, \psi_{1}\right)^{\mathrm{t}}$, and define the pseudospin operators in the $\left(0_{+}, 1\right)$ orbital space,

$$
S_{-\mathbf{p}}^{\mu}=\gamma_{\mathbf{p}} \int d y_{0} \Psi^{\dagger} \frac{1}{2} \sigma^{\mu} e^{i \mathbf{p} \cdot \mathbf{r}} \Psi
$$

with Pauli matrices $\sigma^{a}$ and $\sigma^{0}=1 ; \gamma_{\mathbf{p}}=e^{-\ell^{2} \mathbf{p}^{2} / 4}$. The charge density $\rho_{-\mathbf{p}}=\int d^{2} \mathbf{x} e^{i \mathbf{p} \cdot \mathbf{x}} \psi^{\dagger} \psi$ projected to the $\left(0_{+}, 1\right)$ sector is thereby written as

$$
\bar{\rho}_{-\mathbf{p}}=2\left(w_{\mathbf{p}}^{0} S_{-\mathbf{p}}^{0}+w_{\mathbf{p}}^{3} S_{-\mathbf{p}}^{3}+w_{\mathbf{p}}^{1} S_{-\mathbf{p}}^{1}+w_{\mathbf{p}}^{2} S_{-\mathbf{p}}^{2}\right),
$$

where $w_{\mathbf{p}}^{0}=1-\ell^{2} \mathbf{p}^{2} / 4, w_{\mathbf{p}}^{3}=\ell^{2} \mathbf{p}^{2} / 4, w_{\mathbf{p}}^{1}=i \ell p_{y} / \sqrt{2}$, and $w^{2}(\mathbf{p})=i \ell p_{x} / \sqrt{2}$.

The relevant part of the bilayer Hamiltonian governing the electrons in the $\left(0_{+}, 1\right)$ sector is written as

$$
\Delta \bar{H}=-\int d^{2} \mathbf{x} e A_{0} \bar{\rho}+\int d y_{0} m \Psi^{\dagger} \sigma^{3} \Psi,
$$

where $A_{0}$ stands for an external potential and $m \approx 0.006 \times$ $B[\mathrm{~T}] U$ for the fine level splitting. One can equally write

$$
\begin{aligned}
\Delta \bar{H} & =2 \sum_{\mathbf{p}} \mathcal{P}_{\mathbf{p}}^{\mu} S_{-\mathbf{p}}^{\mu}, \\
\left(\mathcal{P}^{0}, \mathcal{P}^{3}\right) & \approx\left(-m-e A_{0}, m\right),\left(\mathcal{P}^{2}, \mathcal{P}^{1}\right)=\frac{e \ell}{\sqrt{2}}\left(E_{x}, E_{y}\right),
\end{aligned}
$$

where $\mathbf{E}_{\|}=\left(E_{x}, E_{y}\right)=-\partial_{\mathbf{x}} A_{0}$ denotes the in-plane electric field. For the Coulomb interaction one can retain only the intralayer interaction since the pseudo-zero-modes at each valley essentially lie on the same layer,

$$
\bar{H}^{\mathrm{C}}=\frac{1}{2} \sum_{\mathbf{p}} v_{\mathbf{p}}: \bar{\rho}_{-\mathbf{p}} \bar{\rho}_{\mathbf{p}}:
$$

where $v_{\mathbf{p}}=2 \pi \alpha /\left(\epsilon_{\mathrm{b}}|\mathbf{p}|\right)$ with $\alpha=e^{2} /\left(4 \pi \epsilon_{0}\right) \approx 1 / 137$ and the substrate dielectric constant $\epsilon_{\mathrm{b}}$.

\section{Pseudospin textures}

In this section we study the properties of the pseudo-zeromode levels at half filling, using the projected Hamiltonian $\bar{H} \equiv$ $\triangle \bar{H}+\bar{H}^{\mathrm{C}}$, with focus on orbital mixing of the zero-modes.

Let us suppose that the half-filled state $|G\rangle$ is given by a configuration where the pseudospin points in a fixed direction in pseudospin space, i.e., $\left\langle G\left|S_{\mathbf{p}=\mathbf{0}}^{a}\right| G\right\rangle=\frac{1}{2} N_{e} n^{a}$ and $n^{a} n^{a}=1$ with the total number of electrons $N_{e}=2\left\langle G\left|S_{\mathbf{p}=\mathbf{0}}^{0}\right| G\right\rangle$. For $n^{a}$ we use the parametrization $n^{1}=\sin \theta \cos \phi, n^{2}=\sin \theta \sin \phi$, $n^{3}=\cos \theta$, with $-\pi<\theta \leq \pi$ and $0 \leq \phi \leq \pi$.
Note that $n^{3}=1$ corresponds to the filled $n=0_{+}$level while $n^{3}=-1$ represents the filled $n=1$ level. The direction $\mathbf{n}=$ $\left(n^{1}, n^{2}, n^{3}\right)$ would, in general, vary in response to the external field $A_{0}$, and, as $\mathbf{n}$ tilts from $n^{3}= \pm 1$, the $n=0_{+}$and $n=$ 1 levels start to mix. For selfconsistency we assume that $A_{0}$ represents a uniform in-plane electric field $\mathbf{E}_{\|}=-\partial_{\mathbf{x}} A_{0}$ and that it leads to a homogeneous state $|G\rangle$ of uniform density $\rho_{0}=$ $v /\left(2 \pi \ell^{2}\right)$ (with $v=2$ for the spin-degenerate $v=2$ state). We thus consider all configurations with $\left\langle G\left|S_{\mathbf{p}=\mathbf{0}}^{a}\right| G\right\rangle=\frac{1}{2} N_{e} n^{a}$ and single out the ground state or the associated $n^{a}$ by minimizing the energy $\langle G|\bar{H}| G\rangle$.

The calculation of the Coulomb energy $\left\langle G\left|\bar{H}^{\mathrm{C}}\right| G\right\rangle$, in particular, requires the knowledge of pseudospin structure factors $\left\langle G\left|S_{\mathbf{p}}^{\mu} S_{\mathbf{q}}^{v}\right| G\right\rangle$, which, for the present half-filled state with pseudospin $\propto n^{a}$, are given in Ref. [5]. The energy is conveniently written in $\mathbf{x}$ space as $\left\langle G\left|\triangle \bar{H}+\bar{H}^{\mathrm{C}}\right| G\right\rangle=\rho_{0} \int d^{2} \mathbf{x} \mathcal{H}_{\text {eff }}$ with

$$
\begin{aligned}
& \mathcal{H}_{\mathrm{eff}}=-e A_{0}+E(\theta)-\frac{1}{2} V_{1}, \\
& E(\theta)=m(\cos \theta-1)+\mathcal{E} \sin \theta+\frac{1}{32} V_{1}(1-\cos \theta)^{2},
\end{aligned}
$$

where $\mathcal{E}=(e \ell / \sqrt{2}) \mathbf{E}_{\|} \cdot \hat{\mathbf{n}}_{\|}$and $\hat{\mathbf{n}}_{\|}=\left(\hat{n}_{x}, \hat{n}_{y}\right)=(\sin \phi, \cos \phi)$ is an in-plane unit vector;

$$
V_{1}=\sqrt{\pi / 2} \alpha /\left(\epsilon_{b} \ell\right)
$$

sets the scale of the Coulomb exchange energy. In the present notation $\mathcal{H}_{\text {eff }}$ stands for energy per electron in state $|G\rangle$.

Note first that the half-filled pseudo-zero-mode state has an in-plane electric dipole moment

$$
\mathbf{d}_{e}=-\frac{e \ell}{\sqrt{2}}\left(n^{2}, n^{1}\right)=-\frac{e \ell}{\sqrt{2}} \sin \theta \hat{\mathbf{n}}_{\|}
$$

of strength $(e \ell / \sqrt{2})|\sin \theta|$ per electron, proportional to the inplane component $\left(n^{2}, n^{1}\right)$ of the pseudospin. Mixing of the $n=0_{+}$and $n=1$ modes gives rise to this dipole. Note also that the Coulomb correlation energy $\propto V_{1}$ is symmetric about the $n^{3}$ axis $\| \mathbf{B}$. It alone favors $\theta=0$, the filled $n=0_{+}$level, and varies continuously by an amount $\Delta E_{c}=(1 / 8) V_{1}$ as $\mathbf{n}$ sweeps in pseudospin space. The Coulomb interaction thus significantly enhances the splitting of the pseudo-zero-mode levels.

Obviously the energy is lower when when $\mathbf{n}_{\|} \| \mathbf{E}_{\|}$, or $\mathcal{E}=e \ell\left|\mathbf{E}_{\|}\right| / \sqrt{2}$. Accordingly it is convenient, without loss of generality, to suppose that the in-plane field $\mathbf{E}_{\|}$and $\mathbf{n}_{\|}$lie along the $y$ axis, $\mathcal{E}=e \ell E_{y} / \sqrt{2} \geq 0$. With this choice the "1", "2" and " 3 " axes in pseudospin space coincide with the $y, x$ and $-z$ axes in real space, respectively. We henceforth adopt this choice and set $\left(n^{1}, n^{2}, n^{3}\right)=(\sin \theta, 0, \cos \theta)$.

Let us explore stable configurations of the half-filled zeromode state in the next section and here study collective excitations over a given ground state $\left.|G\rangle\right|_{\mathbf{n}}$. We focus on a special class of low-energy collective excitations, pseudospin waves, that are rotations about the energy minimum $\left.|G\rangle\right|_{\mathbf{n}}$ in pseudospin space. Such a collective state is represented as a texture state

$$
|\tilde{G}\rangle=\left.e^{-i O}|G\rangle\right|_{\mathbf{n}},
$$

where the operator $O=\sum_{\mathbf{p}} \gamma_{\mathbf{p}}^{-1} \Omega_{\mathbf{p}}^{a} S_{-\mathbf{p}}^{a}$ locally tilts the pseudospin from $\mathbf{n}$ by small angle $\Omega_{\mathbf{p}}$. The energy $\langle\tilde{G}|\bar{H}| \tilde{G}\rangle=$ 
$\left\langle G\left|e^{i O} \bar{H} e^{-i O}\right| G\right\rangle$ of the excited state is a functional of $\Omega_{\mathbf{p}}^{a}$, and serves as an effective Hamiltonian. The associated Lagrangian is neatly written as

$$
\rho_{0} \int d^{2} \mathbf{x} L_{\mathrm{eff}}=\left\langle G\left|e^{i O}\left(i \partial_{t}-\bar{H}\right) e^{-i O}\right| G\right\rangle .
$$

Actual calculation is made in a systematic way if one notes that the pseudospin operators $S_{\mathbf{p}}^{\mu}$ in Eq. (3) obey the $\mathrm{SU}(2) \times W_{\infty}$ algebra. The result is an effective Lagrangian of the form [5]

$$
\begin{aligned}
L_{\Phi} & =\frac{1}{2}\left(\partial_{t} \Phi\right)^{2}-\frac{1}{2} \Phi\left(M_{\mathbf{p}}\right)^{2} \Phi, \\
M_{\mathbf{p}} & =2 \sqrt{\left(\kappa_{\eta}^{2}+F_{\mathbf{p}}\right)\left(\kappa_{\zeta}^{2}+G_{\mathbf{p}}\right)-\left|W_{\mathbf{p}}\right|^{2}}, \\
\kappa_{\zeta}^{2} & =E^{\prime \prime}(\theta), \quad \kappa_{\eta}^{2}=-\mathcal{E} / \sin \theta,
\end{aligned}
$$

where $\left(F_{\mathbf{p}}, G_{\mathbf{p}}, W_{\mathbf{p}}\right) \sim O\left(V_{1}\right)$ go to zero for $\mathbf{p} \rightarrow 0$. The spectrum $M_{\mathbf{p}}$ of pseudospin waves is in general anisotropic in $\mathbf{p}$ and depends critically on the stable-state configuration $\mathbf{n}$. In particular, $M_{\mathbf{p} \rightarrow 0}=2 \kappa_{\zeta} \kappa_{\eta}$ and $M_{\mathbf{p} \rightarrow \infty} \approx V_{1} / 2+\kappa_{\zeta}^{2}+\kappa_{\eta}^{2}$. With $\theta \rightarrow 0$ and $\mathcal{E} \rightarrow 0$, our $M_{\mathrm{p}}$ precisely reproduces an excitation spectrum derived in Ref. [6] by assuming spatial isotropy.

Note here that the Coulomb interaction alone yields $\kappa_{\eta}^{2}=0$. This implies that, unlike in ordinary bilayer quantum Hall systems, there is no cost of interlayer capacitance energy. Coherence is thus easier to form in the pseudo-zero-mode sector of bilayer graphene.

\section{4. ground states and pseudospin waves}

Let us now examine orbital configurations of the half-filled pseudo-zero-mode state and the associated pseudospin waves. Note first that the Coulomb correlation energy

$$
\Delta E_{c}=\frac{1}{8} \sqrt{\frac{\pi}{2}} \frac{\alpha}{\epsilon_{b} \epsilon_{\mathrm{sc}} \ell} \approx\left(2.2 / \epsilon_{\mathrm{sc}}\right) \sqrt{B[\mathrm{~T}]} \mathrm{meV}
$$

with a typical value $\epsilon_{b} \sim 4$ sets the basic energy scale; here $\epsilon_{\mathrm{sc}}$ effectively takes account of the effect of screening coming from vacuum (Dirac-sea) polarization [5], specific to graphene. In ratio the intrinsic zero-mode level gap $2 m$ and the in-plane field $\mathcal{E}=e \ell\left|\mathbf{E}_{\|}\right| / \sqrt{2} \mathrm{read}$

$$
\begin{aligned}
m / \Delta E_{c} & \approx 3 \epsilon_{\mathrm{sc}} \times 10^{-3} \sqrt{B[\mathrm{~T}]} U[\mathrm{meV}], \\
\mathcal{E} / \Delta E_{c} & \approx 0.9 \epsilon_{\mathrm{sc}} \times 10^{-3} E[\mathrm{~V} / \mathrm{cm}] / B[\mathrm{~T}] .
\end{aligned}
$$

One has to look for the minimum of $E(\theta), E_{\min }=E\left(\theta_{\min }\right)$, to determine the orbital configuration of $|G\rangle$. Let us begin with the case where $\mathbf{E}_{\|}$is absent. (i) For $m>\Delta E_{c}$ (though rather unrealistic), one finds $E_{\min }$ at $\theta=\pi$, i.e., the filled $n=1$ level is realized. The pseudospin waves have a finite energy gap $M_{\mathbf{p}=0}=2\left(m-\triangle E_{c}\right)>0$ and the spectrum is isotropic.

On the other hand, (ii) for $0<m<\Delta E_{c}$, one finds $E_{\min }$ at $\theta= \pm \theta_{\min } ; \theta_{\min }$ varies from 0 to $\pi$ with increasing $m$. Here we encounter a somewhat strange situation: The rotational invariance (about the applied field $\mathbf{B}$ ) is spontaneously broken and the pseudospin waves are gapless.

This actually signals an instability. In this $m$ range the assumed ground state $|G\rangle$, taken to be homogeneous in space,

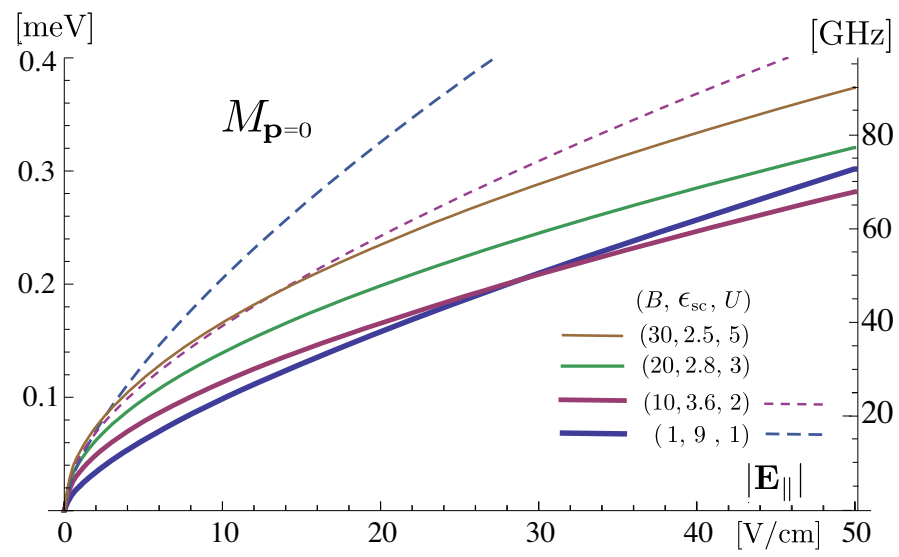

Figure 2: Pseudospin-wave excitation gap $M_{\mathbf{p}=0}$, in units of $\mathrm{meV}$ and $\mathrm{GHz}$, plotted as a function of the in-plane field $\left|E_{\|}\right|$for some typical values of magnetic field $B[\mathrm{~T}]$ and band gap $U[\mathrm{meV}]$. Dashed curves refer to the cases where the screening effect is turned off, $\epsilon_{\mathrm{sc}} \rightarrow 1$.

acquires spontaneous in-plane electric polarization $\propto \sin \theta_{\min }$. Such electrically-polarized homogeneous configurations, unless polarization is relatively weak, are unstable against local charge inhomogeneities and would decay into inhomogeneous configurations. We thus speculate that the half-filled state in the realistic $0<m<\Delta E_{c}$ range may form many domains about local charge excesses for stabilization.

(iii) The in-plane field $\mathcal{E}=e \ell E_{y} / \sqrt{2}>0$ tilts the pseudospin toward $\theta=-\pi / 2$ and competes with $\triangle E_{c}$. The charge carriers thereby acquire a nonzero electric dipole moment $\propto \sin \theta$ and the pseudospin waves always have a finite excitation gap. The potential instability of the texture state, mentioned in (ii), weakens and eventually disappears for large $\left|\mathbf{E}_{\|}\right|$. In Fig. 2 we plot the excitation gap $M_{\mathbf{p}=0}$ as a function of $\left|\mathbf{E}_{\|}\right|$for some typical values of $B[\mathrm{~T}]$ and $m \propto U[\mathrm{meV}] ; M_{\mathbf{p}=0}$ falls in the frequency range of microwaves.

If $\mathbf{E}_{\|}$is sufficiently strong, a sizable gap $M_{\mathbf{p}=0}$ would arise, leading to an incompressible $v=2$ state. One could thereby observe the (spin-degenerate) $v=2$ Hall plateau with a suitably strong injected current. $\mathbf{E}_{\|}$is practically strong for $\mathcal{E} \gtrsim 0.3 \triangle E_{c}$, where $M_{\mathbf{p}=0}=2 \kappa_{\zeta} \kappa_{\eta} \approx 2 \mathcal{E}+\frac{1}{2} \Delta E_{c}$ with $\kappa_{\zeta}^{2} \approx \mathcal{E}+\frac{1}{2} \Delta E_{c}$ and $\kappa_{\eta}^{2} \approx \mathcal{E}$.

It would be a challenge to directly detect the pseudospinwave excitation gap by microwave absorption or reflection experimentally. One may follow Ref. [5] and derive the optical conductance due to transitions within the $\left(0_{+}, 1\right)$ sector,

$$
\begin{aligned}
\Delta \sigma_{x x}(\omega) & =\left(v e^{2} / \hbar\right) \kappa_{\zeta}^{2} \delta\left(\omega-M_{\mathbf{p}=0}\right), \\
\Delta \sigma_{y y}(\omega) & =\left(v e^{2} / \hbar\right)\left(\cos \theta_{\min }\right)^{2} \kappa_{\eta}^{2} \delta\left(\omega-M_{\mathbf{p}=0}\right),
\end{aligned}
$$

which, with disorder taken into account, is significantly peaked around $\omega \sim M_{\mathbf{p}=0}$. Note that the optical conductance is spatially anisotropic, with the larger component $\Delta \sigma_{x x}(\omega)$ perpendicular to $\mathbf{E}_{\|}$(or along an injected current). 


\section{Summary and discussion}

Zero-mode Landau levels, specific to graphene in a magnetic field, deserve attention in their own right. In bilayer graphene they evolve, with a tunable band gap, into two quartets of nearly-degenerate pseudo-zero-mode levels, which, unlike in monolayer graphene, involve two different orbital indices $n=0,1$. One would thus expect interesting quantum phenomena of orbital level mixing.

In this paper we have studied the effects of an external field and the Coulomb interaction on the pseudo-zero-mode quartet. This quartet, especially at half filling, supports, via orbital mixing, quasiparticles with charge and electric dipole, which give rise to characteristic collective excitations, pseudospin waves.

The pseudospin-wave excitation gap $M_{\mathbf{p}=0}$ is generally small, reflecting the intrinsic degeneracy of the pseudo-zero-mode levels, but turns out to depend sensitively on an in-plane field $\mathbf{E}_{\|}$. This means that the gap $M_{\mathbf{p}=0}$ is tunable by an in-plane field or by an injected current.

An experimental signature of the field-induced gap is to observe the quantum Hall effect with an injected current; one would be able to resolve the $v= \pm 2$ Hall plateaus (or the spinresolved $v= \pm 1$ plateaus) using a suitably strong current. A direct study of the excitation gap $M_{\mathbf{p}=0}$ and its field dependence by microwave absorption or reflection would also clarify the unique controllable features of the pseudo-zero-mode sector in bilayer graphene.

This work was supported in part by a Grant-in-Aid for Scientific Research from the Ministry of Education, Science, Sports and Culture of Japan (Grant No. 21540265).

\section{References}

[1] K. S. Novoselov, et al., Nature (London) 438, 197 (2005); Y. Zhang, et al., Nature (London) 438, 201 (2005).

[2] Y. Zheng and T. Ando, Phys. Rev. B 65, 245420 (2002).

[3] E. McCann and V. I. Fal'ko, Phys. Rev. Lett. 96, 086805 (2006).

[4] T. Ohta, A. Bostwick, T. Seyller, K. Horn, and E. Rotenberg, Science 313, 951 (2006);

E. McCann, Phys. Rev. B 74, 161403(R) (2006);

E. V. Castro, et al., Phys. Rev. Lett. 99, 216802 (2007).

[5] T. Misumi and K. Shizuya, Phys. Rev. B 77, 195423 (2008); K. Shizuya, Phys. Rev. B 79, 165402 (2009).

[6] Y. Barlas, R. Côté, K. Nomura, and A. H. MacDonald, Phys. Rev. Lett. 101, 097601 (2008). For earlier discussion of cyclotron resonances in bilayer graphene see also Ref. [7].

[7] D. S. L. Abergel and V. I. Fal'ko, Phys. Rev. B 75, 155430 (2007). 